\title{
Avascular necrosis of the femoral head presenting as trochanteric bursitis
}

\author{
Brian F Mandell
}

\begin{abstract}
Five patients are described with avascular necrosis of the femoral head who presented with ipsilateral trochanteric bursitis, in the absence of clearcut hip joint disease. Avascular necrosis was indicated by magnetic resonance imaging. It is suggested that clinical trochanteric bursitis, especially when refractory to local corticosteroid treatment, may be the initial sign of hip disease. In the patient with risk factor(s) for avascular necrosis that diagnosis should be considered and evaluated with appropriate studies, such as magnetic resonance imaging, to prevent weight bearing at an early stage and permit possible surgical decompression in the hope of postponing or obviating the need for total hip replacement.
\end{abstract}

Patients commonly present to offices, subspecialty clinics, and emergency rooms complaining of 'hip pain'. Many have trochanteric bursitis, a clinical diagnosis which does not require elaborate laboratory or radiographic evaluation. The disorder occurs in healthy active young and old patients, as well as in those with superimposed osteoarthritis of the hip and spine, and patients with rheumatoid arthritis. ${ }^{1-4}$ This condition can be easily and rapidly treated in most cases with one or several local injections of corticosteroids. ${ }^{15}$ It very rarely requires surgery. ${ }^{6}$ Because it is such a common disorder and the diagnosis can be made with fair certainty by palpating point tenderness in the soft tissue overlying the greater trochanter, often totally reproducing the pain, it is not cost effective or medically necessary aggressively to pursue alternative diagnoses in all patients upon initial presentation. As trochanteric bursitis may at times mimic or be associated with hip synovitis, a primary bone process, or radiculopathy, ${ }^{7}$ however, initial careful examination and history as well as follow up are required to rule out these alternative disorders. About $70-90 \%$ of patients respond with decreased or total relief of pain to local injection of anaesthetic and deposit corticosteroid. ${ }^{15}$ This procedure can therefore be considered part of the diagnostic evaluation.

Five cases are discussed in which patients presented with clinical trochanteric bursitis, in the absence of true hip joint disease. They received only transient relief from bursal infiltration with lignocaine and deposit corticosteroid. Follow up evaluations showed new disease referrable to the hip joint in three of the five patients. Because of the presence of risk factors for avascular necrosis, magnetic reson- ance imaging was performed, ${ }^{89}$ and avascular necrosis of the femoral head was diagnosed in all.

\section{Case reports \\ PATIENT 1}

A 23 year old black woman with systemic lupus erythematosus presented on a routine visit with a complaint of pain in the left buttock and lateral thigh. The pain worsened with walking, wearing tight jeans, or sleeping on the left side. Palpation over the left great trochanteric area reproduced the pain, which radiated to just above the knee. There was separate point tenderness to palpation of the ischiogluteal bursa. Systemic lupus erythematosus had been diagnosed four years previously. There had been several previous episodes of bursitis (subacromial) and epicondylitis. She was initially treated with prednisone $40 \mathrm{mg}$ daily, which was gradually reduced over a period of six months. She was lost to follow up for eight months during which time she restarted the prednisone at $20 \mathrm{mg}$ daily. On her return to medical care, about one year before the present visit, the systemic lupus erythematosus was felt to be inactive and steroids were rapidly reduced, then stopped.

A clinical diagnosis of trochanteric bursitis was made and the bursa infiltrated with $1 \mathrm{ml}$ of $2 \%$ lignocaine and $\mathbf{4 0} \mathrm{mg}$ methylprednisolone acetate. Initially, complete relief was obtained, but the pain reoccurred in 10 days. Repeat injection in 21 days, following a repeat examination, which still showed normal hip and knee joints, was also only minimally successful. A third examination 21 days after the second injection showed pain in the area of the trochanteric bursa, and the new onset of groin pain with full internal rotation of the left hip. The gluteal pain had resolved. Magnetic resonance imaging indicated bilateral avascular necrosis. Bone radiographs were normal. The patient refused surgery. Twenty four months after the diagnosis the groin pain with walking persisted, the pain of trochanteric bursitis had mostly resolved, and radiographs showed a crescent lucency on the right and flattening with mild collapse of the femoral head on the left with maintenance of the joint spaces.

\section{PATIENT 2}

A 30 year old white woman who had had systemic lupus erythematosus since the age of 28 , treated previously with high dose corticosteroids, presented with complaints of pain in 
both thighs, right worse than left, exacerbated with walking or lying on either side. Pain on the right side radiated from the trochanter to the lateral knee, pain on the left was specifically localised to the area of the trochanter. Local pressure over the trochanters reproduced the pain. Systemic lupus erythematosus was clinically quiescent with persistent hypocomplementaemia.

Local lignocaine and methylprednisolone acetate injections on both sides relieved the pain on the left, but the pain on the right returned in one week. Examination showed the hips and knees were still normal. Hip radiographs were normal, but computed tomography and subsequent magnetic resonance imaging confirmed the diagnosis of bilateral avascular necrosis. Core decompression was performed on the left and derotational osteotomy on the right. The pathology was recorded as avascular necrosis.

\section{PATIENT 3}

A 33 year old white woman presented with clinical chondromalacia and left anserine bursitis. This was treated locally with injection of lignocaine and prednisolone with total relief. It recurred and was reinjected. Eight months later she presented with lateral thigh pain, worse in the right than the left, worse with walking, and reproduced by firm trochanteric palpation. Hip, spine, and knee examinations were normal. The left trochanteric bursa was injected with lignocaine and methylprednisolone acetate. Initial relief was obtained, but the pain returned within two weeks with additional pain in the right groin. Repeat examination 18 days after the injection showed persistent clinical trochanteric tenderness on both sides, and despite full range of motion there was pain in the right groin with internal and external rotation or abduction. Magnetic resonance imaging showed avascular necrosis on both sides. Plain radiographs showed flattening of the femoral head, greater on the right than the left. Replacement of both hips was planned.

Past medical history was remarkable for idiopathic pseudotumour of the orbit about two years previously. It had been successfully treated with four months of high dose prednisone (80 mg) daily, which was gradually reduced.

\section{PATIENT 4}

A 26 year old asthmatic woman, dependent on steroids (10 mg prednisone daily), presented with right lateral thigh pain radiating to just below the knee. The pain was worse in the morning, worse with walking, and aggravated by lying on the right side in bed. Physical examination showed exquisite trochanteric tenderness to palpation. When the right knee was flexed and the leg cross adducted, severe lateral pain was elicited consistent with fascia lata syndrome. No groin pain was elicited with full external or internal rotation. Local bursal injection and use of a crutch relieved the lateral leg pain, but on re-examination three weeks later there was groin pain with full internal rotation, and decreased but persistent tro- chanteric tenderness to palpation. External rotation and abduction was normal. Magnetic resonance imaging confirmed avascular necrosis on the right side. Use of a crutch and surgical decompression of the femoral head were prescribed, but she was lost to follow up.

\section{PATIENT 5}

A 26 year old black woman with sickle cell anaemia, seen in consultation while in hospital for painful sickle vaso-occlusive crisis, was found to have a swollen right knee. Arthrocentesis showed non-inflammatory $\left(<10^{9}\right.$ white blood cells/l) fluid without crystals. Knee radiographs were normal. Trochanteric bursitis was noted in the same leg and treated with local injection of lignocaine and deposit corticosteroid. There was no evidence of gluteal tendonitis, ischiogluteal bursitis, or hip synovitis on examination. In follow up as an outpatient two weeks later the knee effusion had resolved, but there was persistent upper thigh and new buttock pain with weight bearing, only incompletely reproduced by trochanteric pressure. On full internal rotation pain radiated from the right trochanter to the knee and buttock. Magnetic resonance imaging showed avascular necrosis of both femoral heads and probably the knees. The patient refused surgical intervention and was lost to follow up.

\section{Discussion}

Trochanteric bursitis is a common syndrome, which can usually be relieved by local treatment. Yet, larger series show that there is often an associated hip or back disorder identified clinically or by radiographic studies. In one series of 72 patients with trochanteric bursitis, aged 34-79 years, only $8 \%$ had the bursitis as an 'isolated' condition when radiological studies of lumbar spine and pelvis were performed in all patients. ${ }^{1}$ It is unlikely that the trochanteric bursitis in that series reflected a referred pattern of pain as $90 \%$ of the patients responded with lasting relief to local injection. It would seem more likely that either trochanteric bursitis occurs as a coincident finding in patients having two fairly common disorders or that a primary spine, hip, knee, or foot problem initiates a change in the normal gait, producing increased or altered stress in the area of the fascia lata with resultant trochanteric bursitis or peritrochanteric pain due to gluteal medius tendonitis. Except for patient No 4 there was no clinical suggestion of gluteus tendonitis. As three or fewer local corticosteroid injections give significant improvement in $90-100 \%$ of cases, ${ }^{15}$ lack of response should prompt diagnostic studies for an underlying disorder. Our cases indicate that the 'underlying disorder' may be avascular necrosis of the femoral head. The initial examination and the favourable but nonsustained response to injection of anaesthetic and corticosteroid suggests that the lateral leg pain syndrome described is most consistent with the trochanteric syndrome (including bursitis) rather than due only to referred pain from the avascular necrosis. It would not be cost 
effective to test for avascular necrosis in al patients with risk factors for this disease who present with elinical trochanteric bursitis as some patients with rheumatic diseases (psoriasis, inflammatory bowel disease, lupus, rheumatoid arthritis) treated with corticosteroids also seem predisposed to tendonitis and bursitis.

In a series of renal transplant recipients who developed avascular necrosis the pain was most often described as sudden in onset and localised to the groin, butrock, trochanter, thigh, knee, or low back. Trochanteric tenderness was not consistently found, ${ }^{10}$ and symptoms developed before standard radiographic films indicated avascular necrosis. Zizic et al in a series of 28 patients with systemic lupus erythematosus with avascular necrosis of multiple joints described the presenting complaints as pain on weight bearing or active motion in $77 \%$, and pain at rest in $55 \% .{ }^{11}$ On physical examination pain and limitation of motion were present in $70 \%$ and $52 \%$ of diseased joints respectively, but radiographic findings were already present in most joints at the time of diagnosis. This suggests that those physical findings occur late in the course of the disease process. Colwell has recently described the clinical presentation of avascular necrosis as 'primarily that of pain on weight-bearing that radiates into the groin or knee . . . and a restricted and painful range of motion'. ${ }^{12}$ None of the initial examina- tions of the five patients in this report showed abnormal hip joints, though several patients rapidly developed hip disease. Trochanteric bursitis has not previously been well described as the initial finding in avascular necrosis.

1 Schapira D, Nahir M, Scharf Y. Trochanteric bursitis: a common clinical problem. Arch Phys Med Rehabil 1986; 67 $815-7$

2 Zoltan D J, Clancy W G Jr, Keen J S. A new operative approach to snapping hip and refractory trochanteric bursitis in athletes. Am f Sports Med 1986; 14: 201-4. 3 Raman D, Haslock I. Trochanteric bursitis - a frequent 1982; 41: 602-3.

4 Little $H$. Trochanteric bursitis: a common cause of pelvic girdle pain. Can Med Assoc $\mathcal{F}$ 1979; 120: 457-8.

5 Ege-Rasmussen K J, Fan N. Trochanteric bursitis. Treatment by corticosteroid injection. Scand $\mathcal{F}$ Rheumatol 1985; 14: by corticos

6 Brooker A,F Jr. The surgical approach to refractory trochanteric bursitis. Johns Hopkins Medical foumal 1979 45: 89-100.

7 Swezey R L. Pseudo-radiculopathy in subacute trochanteric bursitis of the subgluteus maximus bursa. Arch Phys Med Rehabil 1976; 57: 387-90.

8 Glickstein M F, Burk D L Jr, Scheibler M L, et al. Avascular necrosis versus other diseases of the hips: sensitivity of $M R$ imaging. Radiology 1988; 169: 213-5.

9 Thickman D, Axel L, Kressel H Y, et al. Magnetic resonance imaging of avascular necrosis of the femoral head. Skeletal Radiol 1986; 15: 133-40.

10 Kenzora J E, Glimcher M J. Avascular necrosis of bone. In Kelley W N, Harris E D Ruddy S, Sledge C B, eds. Textbook of rheumatology. Philadelphia: Saunders, 1981: Textbook

11 Zizic T M, Marcoux C, Hungerford D S, Dansereau J V Stevens M B. Corticosteroid therapy associated with ischemic necrosis of bone in systemic lupus erythematosus. Am F Med 1985; 79: 596-604.

12 Colwell $\mathrm{CW}$. The controversy of core decompression of the femoral head for osteonecrosis. Arthritis Rheum 1989; 32: 797-800. 